\title{
High-permittivity embedded core-shell based nanocapacitors for large scale energy storage
}

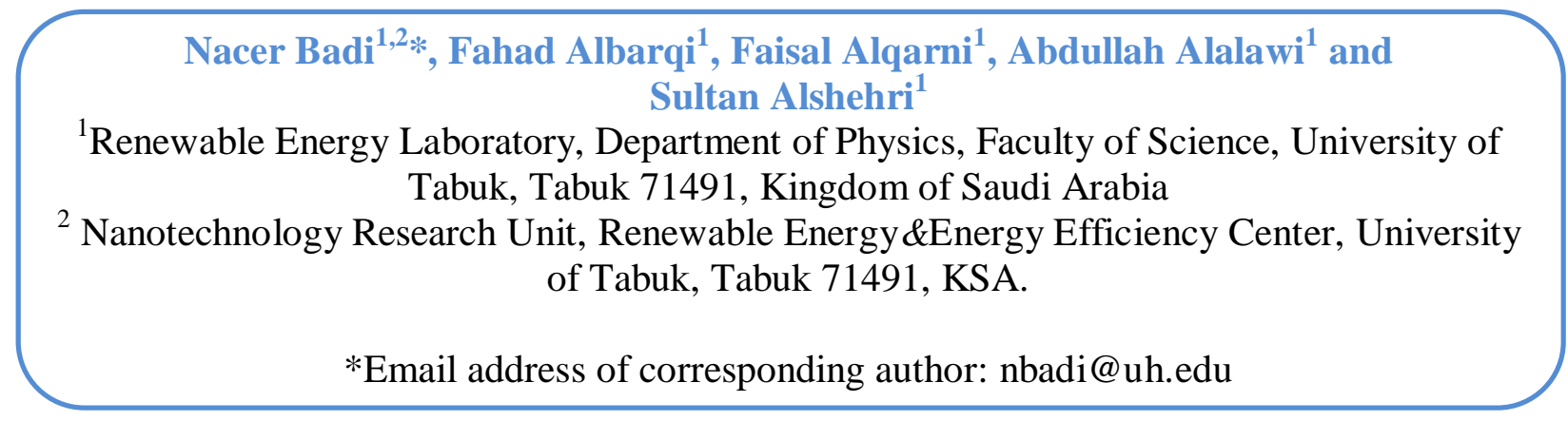

\begin{abstract}
Polymer embedded metal nanofiller is considered as a great choice to achieve high energy storage capacitors. Our earlier experimental work reported that capacitors fabricated with dielectrics consisting of aluminum $(\mathrm{Al})$ core dispersed in highly ferroelectric $\beta$-phase polyvinilydine fluoride(PVDF) polymer, showed an increase in dielectric permittivity $\mathrm{K}$ of the nanocomposite from 12 for pure PVDF polymer to a value of 23.6 when PVDF was embedded with a $20 \%$ volume loading of $\mathrm{Al}-\mathrm{Al}_{2} \mathrm{O}_{3}$ core-shell nanoparticles.In this paper we report on $\mathrm{K}$ values calculation using a modified effective medium theories (EMT). We simulated the system considering less ordered $\mathrm{Al}-\mathrm{Al}_{2} \mathrm{O}_{3}$ nanoparticles in PVDF matrix in order to mimic the experimental configuration of elaborated nanocomposites and so to feedback the experimental work. The AC/DC module is selected in COMSOL Multiphysics software, and in plane electric currents are applied to the physical model. The modified EMT with equations representing the ferroelectric nature of the polymer-based core-shell is used to determine the composite's effective electrical characteristics. The percolation data analysis is utilized to forecast the maximum theoretical $\mathrm{K}$ value, electrical field, and polarization of the nanocomposite including shell interaction. The results of $3 \mathrm{D}$ models with varying filler loadings show a significant increase in electrical permittivity of the composite $(K=2800)$ as compared to electrical permittivity of bare polymer. However, results also indicate a drop in net polarization by a factor of 3 if the embedded core-shells come into contact.
\end{abstract}

Keywords: Nanodielectrics, energy storage, PVDF, core-shell nanoparticles, embedded capacitors

\section{Introduction}

The study of polymer-based nanodielectrics is attracting a lot of attention. Embedded capacitors benefit from such materials because they give more practical energy storage solutions [1-9].Due to their potential uses in energy storage solutions for electronic equipment, the high $\mathrm{K}$ electrical permittivity materials have recently sparked a lot of attention. Capacitors that can hold a significant quantity of charge and distribute it instantly, in particular, are in high demand. This storage capacity is determined by the type of materials used and the polarizability (dipole moment orientation) of the dielectric materials used. This polarizability feature may be increased further by including nanoparticles into 
the polymeric matrix, which can also significantly improve the electrical, thermal, and other mechanical properties of the nanocomposites. Metal-filled polymer composites are of interest in a variety of engineering disciplines. This interest stems from the fact that the electrical properties of such composites are close to those of metals, but the mechanical properties and manufacturing processes are typical of plastics [10].The effect of the type filler and polymer host on the electrical performance of the composite mainly depends on the percolation threshold. This composite overcomes the disadvantage of high loading required for ceramic fillers, but direct contact of conductive fillers will result in a high dielectric loss. This might also form a conductive path in the polymer composites. Therefore, a core-shell structure is proposed for nanofillers, in which an insulating shell is coated around each conducting filler. Non-conductive shells act as inter-particle barriers and prevent the conductive cores from coming in contact with each other during the dispersion process. Core/shell structured nanoparticles can be synthesized using methods such as coating a non-conductive shell on conducting filler. Our approach is to oxidize a conductive metal core to form a nonconductive metal oxide shell around it. Core-shell filler/polymer composites are expected to have high $K$ values because of the increase in net polarization of the dielectric. They will also have low loss tangents due to non-conductive shell. PVDF is now being considered as a great candidate for polymer nanocomposites as it has amazing qualities - it is highly non-reactive to solvents, acids, bases. In the event of fire, PVDF material generates less smoke and it has a low density $\left(1.78 \mathrm{~g} / \mathrm{cm}^{3}\right)$ and low cost compared tothe other fluoropolymers. Apart from the above features it is highly ferroelectric which further helps in enhancing the dielectric response atlower electrical fields with a minimum amountof nanoparticle loading. In this paper, we will use PVDF as a host polymer to make high electrical permittivity (K) material embedded with $\mathrm{Al} / \mathrm{Al}_{2} \mathrm{O}_{3}$ core-shell nanoparticles as shown in Figure 1.

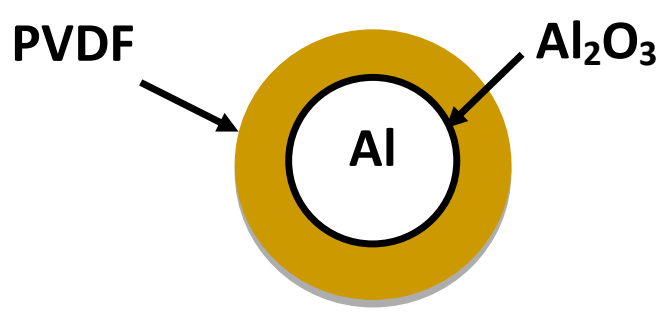

Figure 1. Model of nano-metal/polymer composite.

\section{Background theory}

\subsection{Drude theory}

Debye gave the classical picture of relaxation of polarization with a single relaxation time in a dielectric material [11]. In his work he considered a set of non-interacting dipoles free to rotate in opposition to some viscous resistance in a fluid like medium. The equation for complex permittivity is:

$$
\varepsilon^{*}=\varepsilon_{\infty}+\frac{\varepsilon_{0}-\varepsilon_{\infty}}{1+i \omega \tau}
$$

Where $\varepsilon_{0}=$ Electrical permittivity at low frequency

$\varepsilon_{\infty}=$ Electrical permittivity at high frequency

$\omega=$ Angular frequency

$\tau=$ Relaxation time 
According to Frohlich, the real and imaginary parts ofthe electrical permittivity are given by:

$$
\varepsilon^{\prime}=\varepsilon_{\infty}+\frac{\varepsilon_{0}-\varepsilon_{\infty}}{1+\omega^{2} \tau^{2}}, \varepsilon^{\prime \prime}=\frac{\varepsilon_{0}-\varepsilon_{\infty}}{1+i \omega \tau}(\omega \tau)
$$

$\varepsilon^{\prime}$ determines the polarizability of a material in the presence of an electric field, and $\varepsilon^{\prime \prime}$ determines its intrinsic loss mechanisms [12].

\subsection{Drude Lorentz model}

For metals such as aluminum, complex dielectric function can be decomposed into two components [13-14]. One component is the Drude free-electron term, and the second component is the substantial contribution of the bound or inter-band electrons. Since the dielectric function is additive, it can be written as the sum of free electron and inter-band electron contributions [15] as in Equation 3.

$$
\varepsilon_{\text {bulk }}(\omega)=\varepsilon_{\text {free-electrons }}(\omega)+\varepsilon_{\text {inter-band electrons }}(\omega)
$$

The complex dielectric function of inter-band electrons is calculated by taking into account transitions between $\mathrm{d}$ electrons and conduction sp-band electrons. The imaginary part of bound electron dielectric function arises from inter-band transitions, and the real part arises from polarizability of the bound d-band electrons of aluminum [14]. The expression for the dielectric function of bound electrons can be written using Lorentz oscillator model [13] as shown in Equation 4.

$$
\varepsilon_{\text {int }}=\frac{\omega_{p b}^{2}}{\omega_{0}^{2}-\omega^{2}-i \omega \gamma_{b}}
$$

Where $\omega_{p b}$ is bound electron plasma frequency, $\omega_{0}$ is bound-electron resonant frequency, $1 / \gamma b$ $=T_{\mathrm{b}}$ is the bound-electron decay time and $\omega$ is the angular frequency.

The complex dielectric function for the free electrons is given by Drude model $[13,15]$ as in Equation 5.

$$
\varepsilon_{\text {free }}=1-\frac{\omega_{p f}{ }^{2}}{\omega^{2}+i \omega \gamma_{0}}(5)
$$

Where $\omega_{p f}$ is the free electron plasma frequency and $1 / \gamma 0=\mathrm{T}_{0}$ is the free-electron scattering time. Therefore, bulk electrical permittivity of aluminum can be written as:

$$
\varepsilon_{b u l k}=1-\frac{\omega_{p b}^{2}}{\omega^{2}+i \omega \gamma_{0}}+\frac{\omega_{p b}^{2}}{\omega_{0}^{2}-\omega^{2}-i \omega \gamma_{b}}(6)
$$

For metal particles smaller than their mean free path, decay time has been proved to be particle-size dependent [5].

$$
\gamma_{f}=\frac{1}{T_{0}}+\frac{2 v_{f}}{a}(7)
$$


$\mathrm{V}_{\mathrm{f}}$ is Fermi velocity and $\mathrm{d}$ is the diameter of the particle. Through this modification, size dependencies of the aluminum particles are easily incorporated into its dielectric function expression, which can be written [6] as:

$$
\varepsilon_{(a, w)}=1-\frac{w_{p f}^{2}}{\omega^{2}+i \omega \gamma_{f}}+\frac{\omega_{p b}^{2}}{\omega_{0}^{2}-\omega^{2}-i \omega \gamma_{i}}
$$

Where $\varepsilon(a, \omega)$ is the size dependent electrical permittivity of a metal.

\subsection{Effective Medium Theory}

Effective medium theories are based on the fact that energy differences are easier to calculate than energies. EMTs and other mean-field like theories are physical models based on properties of individual components and their fractions in the composite [16]. Generally, the properties that are calculated using EMTs are dielectric permittivity and conductivity. There are many EMTs, and each theory is more or less accurate under different conditions. Most popular EMTs [12,14] are Maxwell - Garnett model:

$$
\varepsilon_{e f f}=\varepsilon_{h}+3 f\left(\frac{\varepsilon_{i}-\varepsilon_{h}}{\varepsilon_{i}+2 \varepsilon_{h}}\right) \varepsilon_{h(9)}
$$

Symmetric Bruggeman model:

$$
\begin{aligned}
& \varepsilon_{e f f}=\frac{1}{4}\left[3 f\left(\varepsilon_{i}-\varepsilon_{h}\right)+2 \varepsilon_{h}-\varepsilon_{i}+\right. \\
& \left.\sqrt{\left(1-3 f^{2}\right) \varepsilon_{i}{ }^{2}+2\left(2+9 f-9 f^{2}\right) \varepsilon_{i} \varepsilon_{h}+(3 f-2)^{2} \varepsilon_{h}{ }^{2}}\right]
\end{aligned}
$$

Looyenga model:

$$
\varepsilon_{\text {eff }}=\left[\left(\varepsilon_{i}^{\frac{1}{A}}-\varepsilon_{h}^{\frac{1}{A}}\right) f+\varepsilon_{h}^{\frac{1}{A}}\right]^{A}(
$$

Where $\varepsilon_{\text {eff }}$ is the effective electrical permittivity of the medium, $\mathrm{f}$ is the volume fraction of the filler, $\varepsilon_{\mathrm{i}}$ is electrical permittivity of the Au filler, $\varepsilon_{\mathrm{h}}$ is electrical permittivity of the host PVP matrix and A is a depolarization factor, which depends on the shape of inclusions. Value of $A$ is 3 for spherical fillers.

\subsection{Percolation theory}

Percolation theory takes into account the distribution of minor phase in the microstructure of the composite, which depends on its shape, size, and orientation. Percolation theory is one of the easiest mechanisms to model disordered systems because it has little statistical dependency; is an easy concept to realize even for the most complex systems, and its outcomes are realistic for qualitative predictions of random composites [16]. Percolation theory is significant when the loading of the minor phase of the composite (fillers) reaches a critical value, dramatic changes in the electrical characteristics of the system occur, often on the order of more than a hundred times. This critical fraction of filler is called the percolation threshold, $\mathrm{f}_{\mathrm{c}}$. The abrupt changes in the properties of aluminum/PVDF (electrical conductivity) gives us good benchmark to use the percolation theory.A simple power law relation [17] can be used to describe the changes in the system properties near the percolation threshold.

$$
\mathrm{K}_{\mathrm{h}}\left|f-f_{c}\right|^{-\mathrm{s}}(12)
$$


Where $\mathrm{K}$ is the effective dielectric permittivity, $\mathrm{Kh}$ is the dielectric permittivity of the host $\mathrm{PVDF}$ material, $\mathrm{f}$ is the fraction of inclusions and $\mathrm{f}_{\mathrm{c}}$ is the fraction of inclusions at the percolation threshold and $\mathrm{s}$ is an exponent of value 1 for metal fillers.

\section{Use of COMSOL Multiphysics}

Effective properties of the nanocomposites can be calculated using COMSOL Multiphysics, a finite element method (FEM) based simulation software. FEM often known asfinite element analysis (FEA), is a numerical technique used to find approximate solutions of partial differential and integral equations arising from engineering and physics problems FEM requires a problem to be defined in geometry and subdivided into a number of symmetrical identities called as mesh elements. The effective medium theory and expanded effective medium theory, as well as other related mean field theories [18], were employed. To compute the effective permittivity, the EMT uses different parameters of the resulting medium such as form, size, proportion of inclusions, individual dielectric permittivity, and so on to calculate the effective permittivity. The complex dielectric permittivity of PVDF is calculated using the Drude theory and the one for the metal is calculated using DrudeLorentz model. The fractions of inclusions were taken from a minimum of 0 to a maximum of 1. Percolation theory helps in determining the system behavior near percolation threshold, which is the essential characteristic of the system, and it is studied using a simple power law expression.

The primary goal of simulation in this research is to calculate the effective permittivity of the composite medium formed by PVDF and $\mathrm{Al}_{2} \mathrm{O}_{3}$ core-shell nanoparticles. Effective permittivity can be modeled using percolation theory and effective medium theories (EMT). In plane electric current model was used to simulate the effective properties of nanofiller/polymer dielectric composite capacitors.

\subsection{Creating geometry}

First step towards a successful FEM simulation is to create a geometry that is most suitable to the problem being solved. Geometries needed for simulation of the composite nanodielectric environment are drawn in 3D models with varying filler fractions. Each loading fraction in eithermodel is created as a separate file with an independent geometry.Figure 2 shows the geometry setup of 3D modelnanodielectric embedded coreshell nanoparticles with spherical disks of core radius $35 \mathrm{~nm}$ and shell radius $45 \mathrm{~nm}$ that are enclosed in a rectangular block of fixed area that defines the PVDF polymer matrix. The location of different nanoparticle is randomly generated by using a MATLAB function and the corresponding geometry is built in COMSOL software.

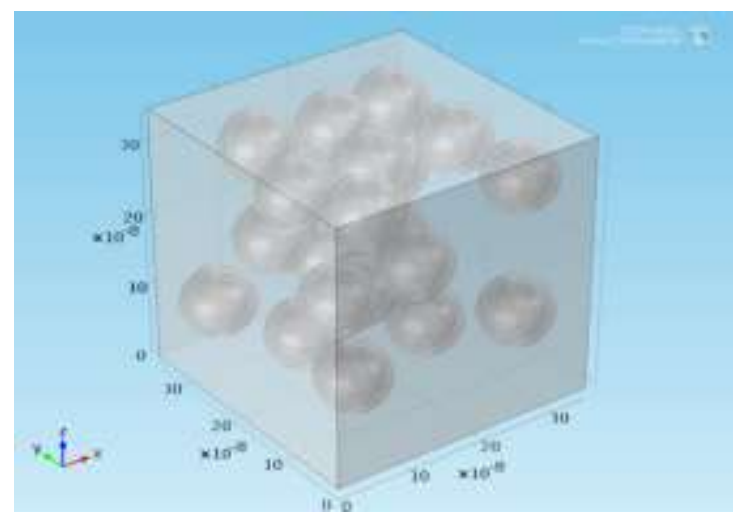

Figure 2. Polymer matrix with randomly dispersed nanoparticles. 


\subsection{Setting the model}

In COMSOL Multiphysics, Electrostatics -in-plane electric current module was used to simulate the effective properties of $\mathrm{Al} / \mathrm{Al}_{2} \mathrm{O}_{3}$ core-shell nanofiller/PVDF composite capacitors. This module was selected because it allows a frequency sweep to be conducted for different loadings of the nanofiller at different frequencies. In sub-domain settings, there is a provision to choose the basic governing equation; polarization $\mathrm{P}$ which is directly proportional to the applied electric field $\mathrm{E}$ is given by: $\mathrm{P}=\varepsilon_{\mathrm{o}} *\left(\varepsilon_{\mathrm{r}}-1\right) *$ Eof the composite, which are the major concepts of the governing physics of metal-insulator composite nanodielectric capacitors. Therefore, these concepts are primarily considered in the analysis of simulation results.

After the geometry is drawn, each section of it is assigned to appropriate materials using subdomain settings. In a 3D geometry, topface is set to the port where input alternating voltage (sine function is considered in simulations with unit amplitude) is applied, while its opposite face is maintained at ground. The remaining sides are maintained at periodic condition. This makes the geometry analogous to a parallel-plate capacitor with a voltage applied across its plates.

\subsection{Solving the model}

The final step before simulating the modelafter creating the geometry, declaring its physical properties and defining its guiding equations and constants, is setting the variables. For this, a parametric solver is used in COMSOL Multiphysics. The solution is obtained from simulations by setting the variables and their possible values through parametric solver. This completes the model building after which it is solved and analyzed.

\section{Results and Discussions}

\subsection{Electrical permittivity of PVDF}

The complex electrical permittivity of PVDF material as explained is given by Drude theory from above mentioned equations and considering the following: damping term given by $\log \Gamma=-16.41$ and electrical permittivity $\left(\xi_{0}\right)=12$ at low frequency and $\left(\xi_{00}\right)=4.5$ at higher frequencies.Figures $3 \mathrm{a}$ and $3 \mathrm{~b}$ show the imaginary and real plot of electrical permittivity of PVDF material in accordance with Drude theory, respectively.

a

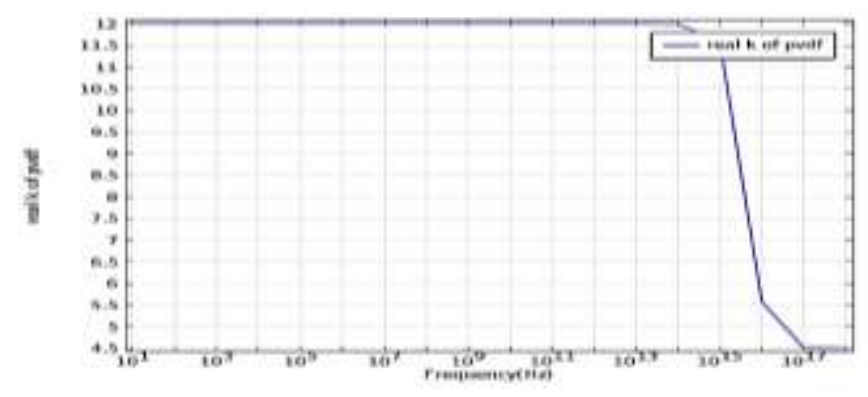

b

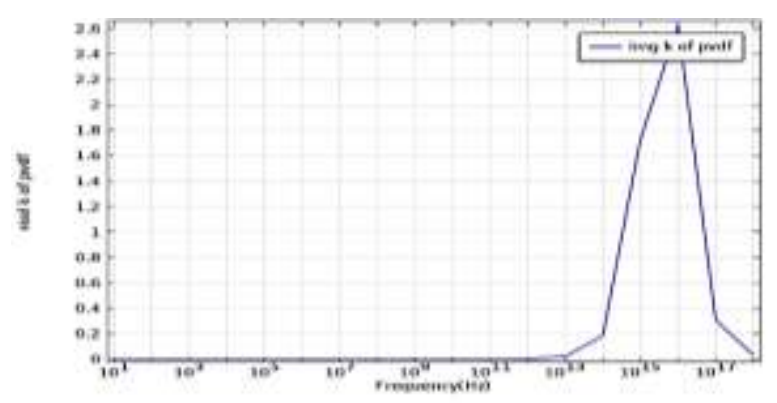

Figure 3. Real part (a) and imaginary part (b) of electrical permittivity of PVDF

\subsection{D Modeling}

In order to determine the characteristics in 3D model, three geometries were drawn with loadingfractions of $0.03,0.12,0.1575$ corresponding to $4,16,21$ spheres. The radii of the $\mathrm{Al}$ disks are considered to be $35 \mathrm{~nm}$ and that of the shell to be around $45 \mathrm{~nm}$ which are enclosed in a cube (polymer matrix) of side $800 \mathrm{~nm}$. When an alternating voltage of 1 volt is applied, 
an electric field is generated in the dielectric using the boundary conditions. Figure 4 shows the slice plots of electric field Distribution in the 3D dielectrics with nanofillers of fraction: $0.03,0.12$, and 0.1575 . The highest enhancement of the electric field is observed inthe samples with loadingsvalues closeto the percolation threshold. The net electrical field of the composite increases with loading and results in a highnet polarization. The corresponding loading fraction is close to the percolation threshold $\mathrm{f}_{\mathrm{c}}=0.16$. Figure 5 shows the isosurface plots of the polarization in the $3 \mathrm{D}$ dielectrics with nanofillers of fraction: $0.03,0.12$, and 0.1575 .In the presence of electric field, there are two polarizations acting on the medium. The first is the polarization of the PVDF matrix and the second one is the local polarization due to the Al fillers. These two polarizations contribute to the net effective polarization. With an increase in net polarization, there is also increase in electrical permittivity of the composite.

a

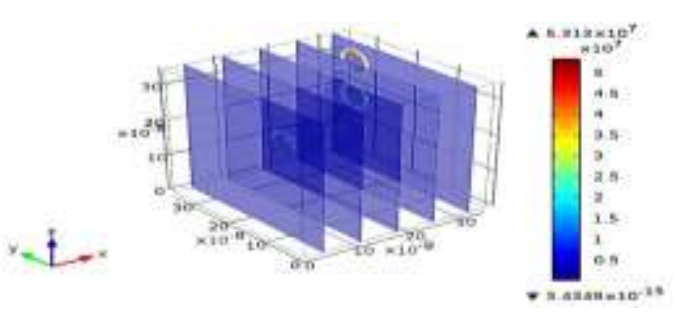

b

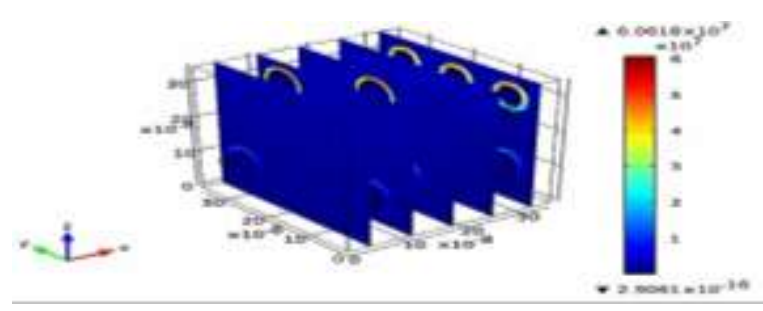

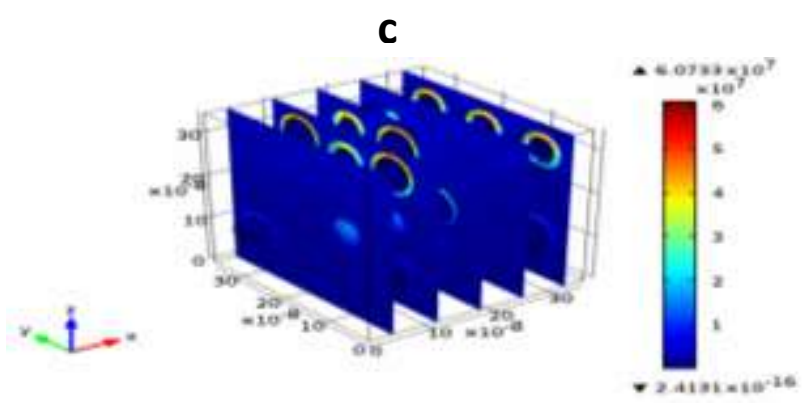

Figure 4. Electric field generated in nanodielectric with a) $f=0.03 \mathbf{b}) f=0.12 \mathbf{c}) f=0.1575$
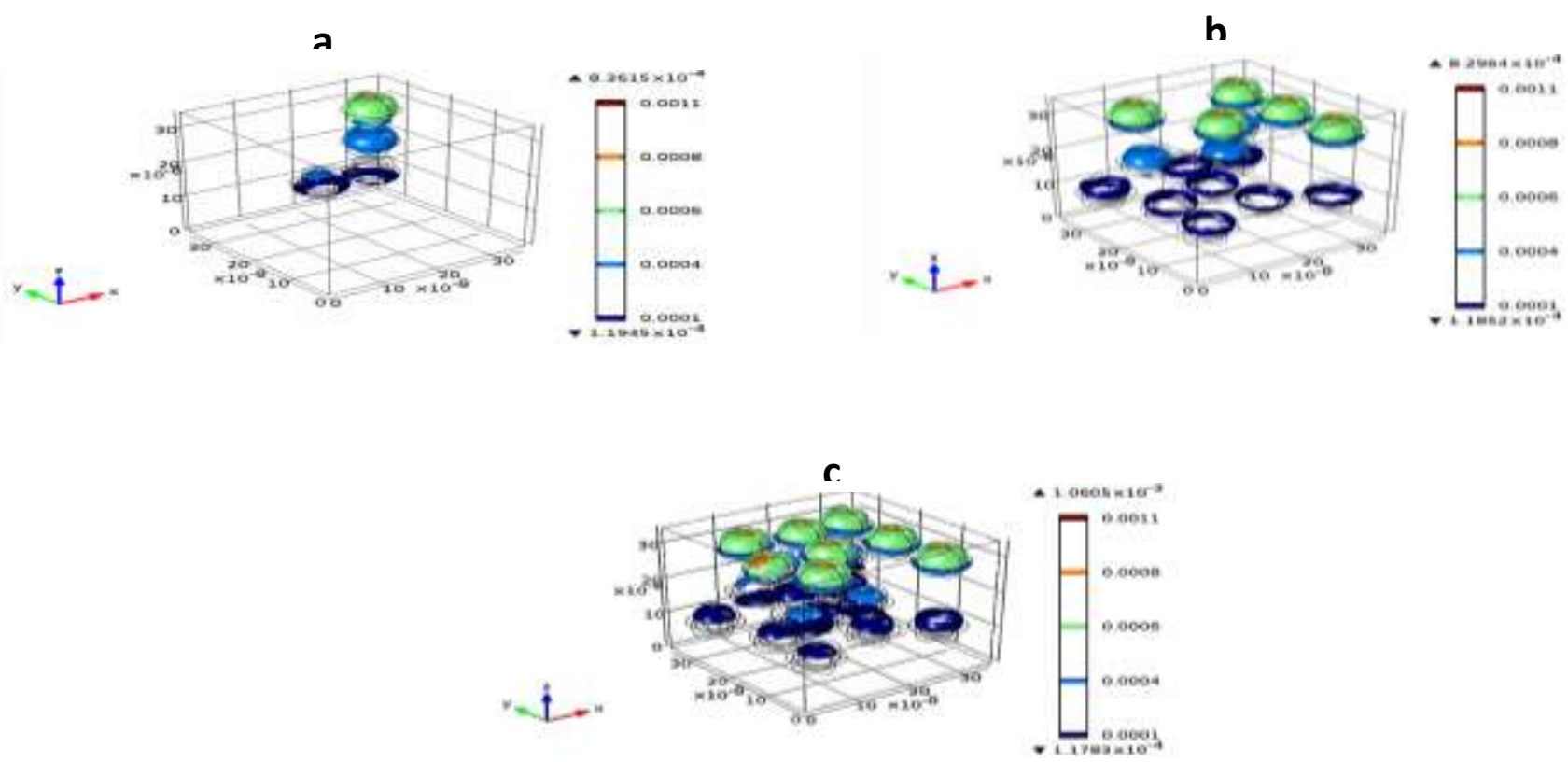

Figure 5. Polarization generated in nanodielectric with a) $f=0.03 \mathbf{b}) f=0.12$ c) $f=0.1575$ 


\section{4. $\mathrm{K}$ value calculation using EMTs}

The $\mathrm{K}$ value of the medium is calculated using the effective medium theory equations 9 and 10.

In Figures 6a and 6b, both Maxwell Garnett and Symmetric Bruggeman models gave an electrical permittivity of the composite in good agreement with the experimental values we obtained in a similar composite at the same loading. The effective electrical permittivity decreases at higher frequencies which is due to decay of ionic and polarization.

a

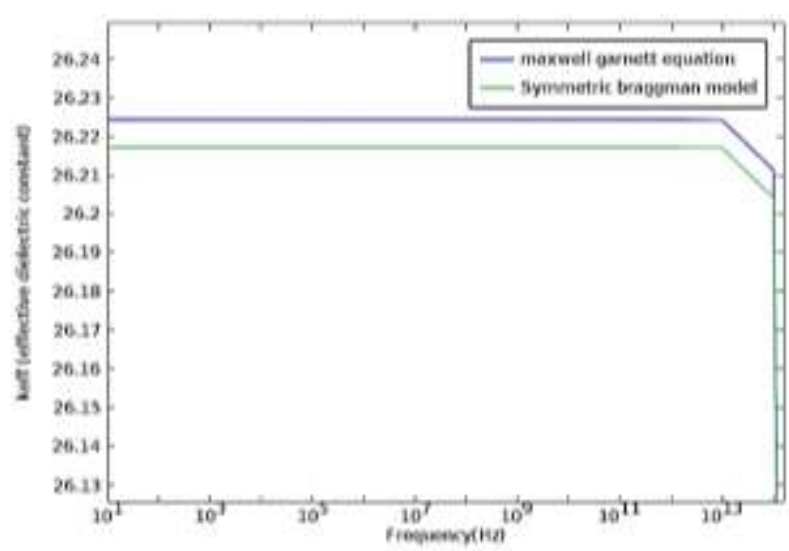

b

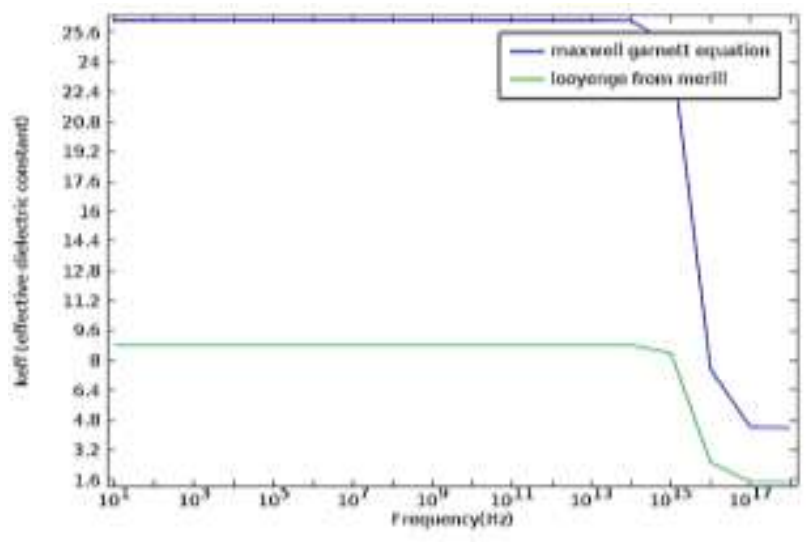

Figure 6. EMT plot at a loading $\mathrm{f}=0.1575$ for a) Maxwell Garnett and symmetric Bruggeman model and b) Maxwell Garnett and Looyenga model

\subsection{Percolation Threshold}

The technique of preparing percolative composites to increase electrical permittivity $(\mathrm{K})$ of the polymer- based capacitors greatly depends on the concentration of the nanofiller. As mentioned earlier, $\mathrm{K}$ value of composites can be dramatically increased when loading of the nanofillers is in the vicinity of the percolation. Figure 7 shows an increase of permittivity from 12 of bare PVDF to 2800 of the nanocomposite at percolation threshold $\mathrm{f}=0.16$.

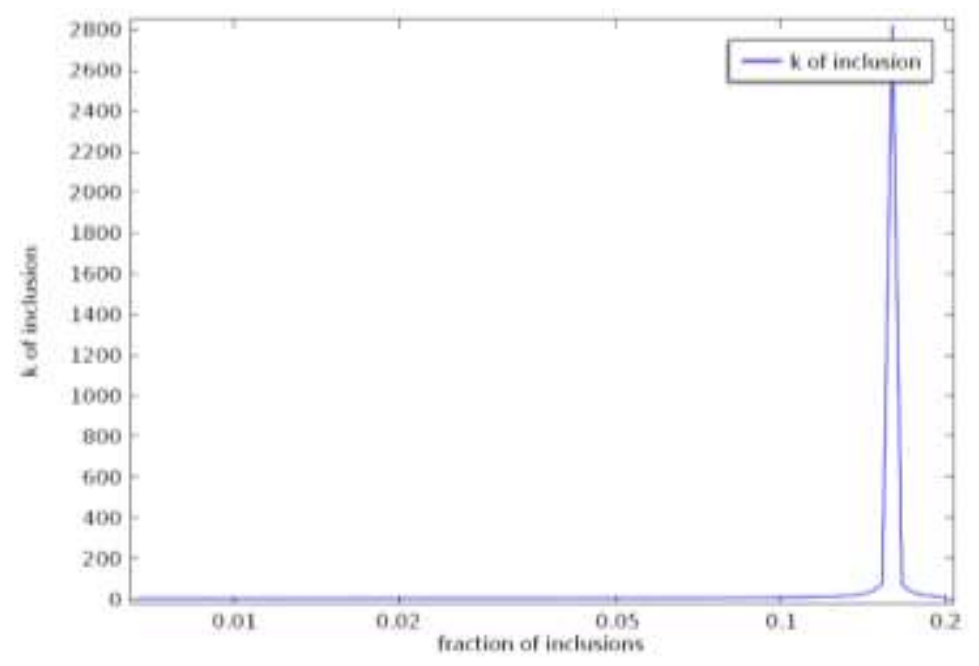

Figure 7. Calculated electrical permittivity of the composite versus nanofiller loading using equation 12 


\subsection{Case study at the interaction of two core-shells}

We also investigated the close interaction of the core-shell nanoparticles dispersed in polymer and its effect on the net polarization. As expected with the use of a capping shell for electrical insulation of metal cores, the simulation results shown in Figure 8 indicate a drop in net polarizationby a factor of 3 at the contact region. This may explain the low value of electrical permittivity obtained $(\mathrm{K}=23.6)$ at a loading of $20 \%$ as earlier reported in our experimental work [1]. Due to their surface energy, nanoparticles agglomerate easily and form electrically conductive paths within the composite. Therefore, dielectric permittivity can only be enhanced through well dispersed nanoparticles within the polymer.

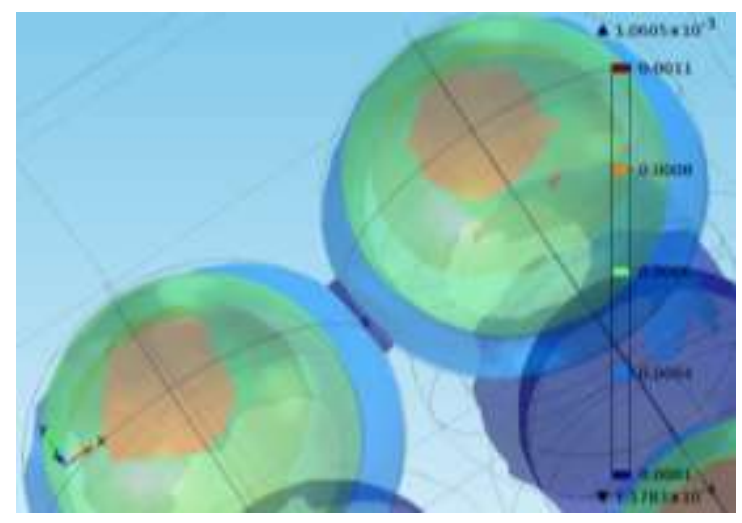

Figure 8. Polarization variation at the interaction of alumina shells

\section{Conclusion}

Complex electrical permittivity of PVDF is calculated using Drude theory and that of aluminum using Drude-Lorentz model. Enhancement of local and net polarization, electric field is observed with increasing loading of nanoparticles in polymer till percolationthreshold is reached. The above case is studied for 3D models, and graphs were plotted to explain the phenomenon of percolation. Significant increase in electrical permittivity of the composite $(\mathrm{K}=2800)$ is achievedwhen compared to electrical permittivity of bare polymer $(\mathrm{K}=12)$ by introducing $35 \mathrm{~nm}$ sized Al nanoparticles. Effective electrical permittivity of the composite was also calculated using EMTs of Maxwell-Garnett, symmetric Bruggeman and looyenga model. Like the percolation theory, EMTs also predict an increasing trend of electrical permittivity with an increase in volume fraction of the filler. Also, variation of polarization near the shell interaction is studied and plotted.A drop in net polarization by a factor of 3 is observed if the embedded core-shells come into contact.This may explain the low value of electrical permittivity obtained $(\mathrm{K}=23.6)$ at a loading of $20 \%$ as earlier reported in our experimental work [1]. Due to their surface energy, nanoparticles agglomerate easily and form electrically conductive paths within the composite. Therefore, dielectric permittivity can only be enhanced through well dispersed nanoparticles within the polymer.

\section{Acknowledgments}

The authors would like to acknowledge financial support for this work, from the Deanship of Scientific research (DSR), University of Tabuk, Tabuk, Saudi Arabia, under Grant No. S-0193/1441.

\section{Authors contribution}

The manuscript was written through contributions of all authors. All authors have given approval to the final version of the manuscript. 


\section{Compliance with ethical standards}

\section{Conflict of interest}

The authors declare that they have no conflict of interest.

\section{References:}

1. Nacer Badi; Rakesh Mekala; Syed Khasim; Aashis Roy; Alex Ignatiev, "Enhanced dielectric performance in $\mathrm{PVDF} / \mathrm{Al}-\mathrm{Al}_{2} \mathrm{O}_{3}$ core-shell nanocomposites", Journal of Materials Science 29, 10593 (2018).

2. C. Tang, G. Long, X. Hu, K.W. Wong, W.M. Lau, M. Fan, J. Mei, T. Xu, B. Wang, D. Hui, Conductive polymer nanocomposites with hierarchical multi-scale structures via self-assembly of carbon-nanotubes on graphene on polymer-microspheres. Nanoscale. 6 , 7877 (2014)

3. G. Keledi, J. HJri, B. PukJnszky, Polymer nanocomposites: structure, interaction, and functionality. Nanoscale. 4, 1919 (2012).

4. L. Zhu, Q. Wang, Novel ferroelectric polymers for high energy density and low loss dielectrics. Macromolecules. 45, 2937 (2012).

5. J. Ho, R. Jow, Characterization of high temperature polymer thin films for power conditioning capacitors, DTIC Document (2009).

6. Q. Chen, Y. Shen, S. Zhang, Q. Zhang, Polymer-based dielectrics with high energy storage density. Annu. Rev. Mater. Res. 45, 433-458 (2015).

7. S. Wu, W. Li, M. Lin, Q. Burlingame, Q. Chen, A. Payzant, K. Xiao, Q. Zhang, Aromatic polythiourea dielectrics with ultrahigh breakdown field strength, low dielectric loss, and high electric energy density. Adv. Mater. 25, 1734 (2013).

8. M. Sindu Shree, H. Schulz-Senft, N.H. Alsleben, Y.K. Mishra, A. Staubitz, R. Adelung, Light, force, and heat: a multi-stimuli composite that reveals its violent past. ACS Appl. Mater. Interfaces. 9, 38000 (2017).

9. Z.M. Dang, M.S. Zheng, J.W. Zha, (2016) 1D/2D carbon nanomaterial-polymer dielectric composites with high permittivity for power energy storage applications. Small 12, 1688.

10. J.K. Nelson, J.C. Fothergill, Internal charge behavior of nanocomposites. Nanotechnology. 15, 586-595 (2004).

11. P Debye "Polar Molecules" Chemical Catalogue Co NY (1929).

12. N.K. Grady, N.J. Halas, P. Nordlander, "Influence of dielectric function properties on the optical response of plasmon resonant metallic nanoparticles", Chemical Physics Letters 399, 167-171, 2004.

13. A. E. Neeves and M.N.Birnboim, "Composite Structure for the enhancement of nonlinear-optical susceptibility", J.Opt Soc. Am. B. Vol 6, No. 4, April 1989.

14. R. D. Averitt, D. Sarkar, and N. J. Halas, "Plasmon Resonance Shifts of Au-Coated Au2S Nanoshells: Insight into Multicomponent Nanoparticle Growth", Phys Rev Lett, Vol 78 No 22, June 1997.

15. Lucia B Scaffardi and Jorge O Tocho, "Size dependence of refractive index of gold nanoparticles", Institute Of Physics Publishing, Nanotechnology, 17, 1309-1315, 2006.

16. C. W. Nan, Y. Shen and Jing Ma, "Physical properties of composites near percolation", Annu. Rev. Mater. Res. 2010. 40:3.1-3.21

17. Geoffrey Grimmett, "Percolation", Springer link, 1991.

18. V. Myrochnychenko and C. Brosseau, "Finite-element method for calculation of the effective permittivity of random inhomogeneous media", Physical Review E 71, 016701, 2005. 